\title{
Optimal Vaccination, Treatment, and Preventive Campaigns in Regard to the SIR Epidemic Model
}

\author{
E.V. Grigorieva ${ }^{*}$, E.N. Khailov ${ }^{2}$ \\ ${ }^{1}$ Department of Mathematics and Computer Sciences, Texas Woman's University, \\ Denton, TX 76204, USA \\ 2 Department of Computational Mathematics and Cybernetics, Moscow State Lomonosov University, \\ Moscow, 119992, Russia
}

\begin{abstract}
The Susceptible-Infected-Recovered (SIR) model for the spread of an infectious disease in a population of constant size is considered. In order to control the spread of infection, we propose the model with four bounded controls which describe vaccination of newborns, vaccination of the susceptible, treatment of infected, and indirect strategies aimed at a reduction of the incidence rate (e. g. quarantine). The optimal control problem of minimizing the total number of the infected individuals on a given time interval is stated and solved. The optimal solutions are obtained with the use of the Pontryagin Maximum Principle and investigated analytically. Numerical results are presented to illustrate the optimal solutions.
\end{abstract}

Keywords and phrases: SIR model, control the spread of infection, nonlinear control system, Pontryagin maximum principle, Riccati equation, generalized Rolle's theorem

Mathematics Subject Classification: 49J15, 58E25, 92D30

\section{Introduction}

Mathematical epidemiology can be traced to 1760 when Daniel Bernoulli stated and solved a model for the spread of smallpox. Thereafter, epidemiology developed slowly as established mathematicians did not show much of interest in application problems. Only in 1906 when Hamer published a discrete model of the spread of measles did epidemiology become resurgent. Hamer was followed in 1922 by Ronald Ross who modeled malaria and how it can be spread by mosquitos bites. Finally, when the physician Kermack and biologist McKendrik (1927) added to the standard epidemic model the rates of mortality and rates of birth, it can be claimed that epidemic models were firmly established as applications of interest in the mathematical literature. With the appearance of AIDS and HIV in 1985, mathematical epidemiology became a tool of public concern. An excellent review of the history of mathematical epidemiology can be found in the monograph [28].

Much of the literature of mathematical modeling of epidemics has been published by experts in the biological sciences and suggest models that defy solution by current analytical mathematical methods. Of the thousands of papers and books on epidemics, several of the most outstanding are [1,3]. However, not many papers are devoted to the control of epidemics. Although "control" is mentioned as an objective

\footnotetext{
${ }^{*}$ Corresponding author. E-mail: egrigorieva@mail.twu.edu
} 
of many papers, the meaning of control is most often as the simulation of a model under parameter sets such that the reproduction ratio is reduced below unity $[1,6,28]$. There has been very little published in the application of optimal control theory to epidemiology. Systematic application of the optimal control theory to epidemiology has its beginnings in the late 70 s with some important results published by $[2,7,8,16,18,19,22,26,31,32]$.

Optimal control is characterized by the use of the Pontryagin Maximum Principle. For example, in [31] the Kermack-McKendrick epidemic model is investigated in respect to the Bellman equation, and in $[21,25]$ the authors solve the optimality system numerically as the original state system coupled with the adjoint system. The latter method is called a two-point boundary value problem (TPBVP) for the Maximum Principle. While methods of numerical solution of this problem are well developed, we emphasize analytical methods and demonstrate how the type of optimal control can be obtained through qualitative investigation of the switching functions. We refer the reader to the works of $[4,7,23]$ that use analytical methods of optimal control and study control models qualitatively.

In [23], the authors analytically study an optimal control problem of a general Susceptible-InfectedRemoved (SIR) model with two bounded controls: vaccination and treatment. In [4] different epidemic models with a general type of interaction between susceptible and infected species with one temporal control is considered. The goal of [7] is to investigate how the optimal start of educational campaigns depend on the time duration of the campaign. In $[4,7,23]$ the objective is to minimize the cost of epidemics.

Our paper deals with an optimal control of a SIR type epidemic model with four simultaneous bounded controls. We state an optimal control problem of minimizing the cumulative (integral) level of the infective individuals on a given time interval. The advantages of an analytical investigation are: a warranty is provided that the type of optimal solution can be found for any given set of the model parameters, a complex TPBVP for the Maximum Principle can be reduced to a considerably simpler problem of finite dimensional optimization, and relevant guidance for the practical design of control policy becomes transparent.

The paper is organized as follows. In Section 2, we introduce the model, simplify it, and then study some qualitative properties of its solutions. In Section 3, the optimal control problem with four bounded controls in which we seek to minimize the cumulative number of the infected individuals on a given time interval is stated. The existence and analysis of the corresponding optimal solution is investigated using the Pontryagin Maximum Principle. Then the adjoint system of the corresponding TPBVP for the Maximum Principle is transformed into a non-autonomous homogeneous system of linear differential equations for the switching functions which entirely determine the types of the optimal controls. In Section 4 a detailed analysis of solutions of the aforementioned system of differential equations is presented. This analysis identifies two different cases dependent on the parameters of the simplified model. In the first case the optimal controls are constant functions. In the second case, along with the optimal controls that are constant functions, there is an optimal control which is a piecewise constant function with one moment of switching. Our analytical investigation allows to reduce a complex TPBVP for the Maximum Principle to the one-dimensional constrained optimization problem, which replaces the original optimal control problem and simplifies its solution. A detailed description of the approach employed for solving the considered problem as well as the results of our numerical calculations and their analysis are presented in Section 5. Finally, in Section 6 our conclusions are given. 


\section{Description of the Model}

We consider the Cauchy problem on the time interval $[0, T]$ for a nonlinear control system of differential equations:

$$
\left\{\begin{array}{l}
\dot{S}(t)=\mu N(1-p(t))-\frac{\beta}{1+r(t)} S(t) I(t)-(\mu+q(t)) S(t), t \in[0, T], \\
\dot{I}(t)=\frac{\beta}{1+r(t)} S(t) I(t)-(\gamma+\mu+s(t)) I(t), \\
\dot{R}(t)=-\mu R(t)+q(t) S(t)+(\gamma+s(t)) I(t)+\mu N p(t), \\
S(0)=S_{0}, I(0)=I_{0}, R(0)=R_{0} ; S_{0}, I_{0}, R_{0}>0 .
\end{array}\right.
$$

which describes the spread of an infectious disease in a population. This population of a constant size $N$ is divided into three sub-populations: that of susceptible individuals, of size $S(t)$, of infected individuals, of size $I(t)$, and of removed (recovered and immune) individuals, of size $R(t)$. It is usually assumed that the population size is constant (that is, the demographic processes are considerably slower than the epidemic ones), that all newborns are susceptible and hence enter compartment $S$, and that a recovery implies permanent immunity. Unfortunately, "removed" can stand for both, treated or recovered and those individuals who died. In system (2.1), the values $\mu$ and $\gamma$ are the birth-death rate and recovery rate, respectively; and $\beta$ is the disease transmission rate. The functions $p(t), q(t), r(t)$ and $s(t)$ are controls: $p(t)$ is the vaccination rate of the newborns; $q(t)$ is the vaccination rate of the susceptible; $s(t)$ is the rate at which infectious individuals are treated and $r(t)$ is the impact of indirect strategies or precocious actions such as quarantine, wearing masks, closing public events, etc. Term $s(t) I(t)$ is similar to one in work of [23] and it means the cure of infected individuals due to a medical treatment. Additionally, similar to $[4,7]$ we intend to reduce the incidence rate by controlling a transmission rate. As in [4] we replace coefficient of the product of $S(t)$ and $I(t)$ by a decreasing function of control. However, while [4] does not specify this function, we believe that the transmission rate coefficient must be inversely proportional to the control $r(t)$, and in our model it is given by the expression $\beta(1+r(t))^{-1}$. Moreover, while in $[4,7]$, control is responsible for health-promotional campaigns, in our paper, the control $r(t)$ has a broader meaning, that is all possible indirect strategies aimed to reduce the incidence rate such as methods of isolation, quarantine, health promotional campaign, screening, and even wearing masks, etc.

Our control functions $p(t), q(t), r(t)$ and $s(t)$ satisfy the restrictions:

$$
\begin{array}{ll}
0 \leq p(t) \leq 1, & 0 \leq q_{\min } \leq q(t) \leq q_{\max }<1, \\
0 \leq r_{\min } \leq r(t) \leq r_{\max }, 0 \leq s_{\min } \leq s(t) \leq s_{\max }<1 .
\end{array}
$$

The values $S_{0}, I_{0}, R_{0}$ are the positive initial conditions satisfying the equality

$$
S_{0}+I_{0}+R_{0}=N
$$

and values $\mu, \beta, \gamma, N$ and $T$ are positive constants.

The constant population size assumption implies that

$$
S(t)+I(t)+R(t)=N, \quad t \in[0, T],
$$

and hence the system (2.1) can be reduced to two equations.

Let denote in system (2.1) the total birth rate by $\lambda=\mu N$, and introduce the new control functions:

$$
u(t)=\mu+q(t), v(t)=\beta(1+r(t))^{-1}, w(t)=1-p(t), \quad \eta(t)=\mu+\gamma+s(t),
$$

satisfying the restrictions:

$$
\begin{array}{ll}
0<u_{\min } \leq u(t) \leq u_{\max }, & 0<v_{\min } \leq v(t) \leq v_{\max }, \\
0 \leq w(t) \leq 1, & 0<\eta_{\min } \leq \eta(t) \leq \eta_{\max },
\end{array}
$$

where

$$
\begin{aligned}
& u_{\min }=\mu+q_{\min }, \quad u_{\max }=\mu+q_{\max } ; \\
& v_{\min }=\beta\left(1+r_{\max }\right)^{-1}, \\
& s_{\min }=\mu+\gamma+s_{\min }, \quad s_{\max }=\mu\left(1+r_{\min }\right)^{-1} ;
\end{aligned}
$$


Then, the system (2.1) can be written as the following nonlinear control system of differential equations:

$$
\left\{\begin{array}{l}
\dot{S}(t)=\lambda w(t)-v(t) S(t) I(t)-u(t) S(t), t \in[0, T] \\
\dot{I}(t)=v(t) S(t) I(t)-\eta(t) I(t) \\
S(0)=S_{0}, I(0)=I_{0} ; S_{0}, I_{0}>0
\end{array}\right.
$$

For this control system, all possible Lebesgue measurable functions $(u(t), v(t), w(t), \eta(t))$, which for almost all $t \in[0, T]$ satisfy the inequalities (2.5), form the set of admissible controls $\Omega(T)$.

The positiveness, boundedness and extensibility of solutions $S(t), I(t)$ to the system (2.6) are given by the following lemma.

Lemma 2.1. Let $(u(\cdot), v(\cdot), w(\cdot), \eta(t)) \in \Omega(T)$ be arbitrary controls. Then, the corresponding solution $(S(t), I(t))$ of the system (2.6) are defined on the entire interval $[0, T]$ and satisfy the inequalities:

$$
S(t)>0, \quad I(t)>0, \quad S(t)+I(t)<N, \quad t \in[0, T] .
$$

Proof. For the given controls $(u(\cdot), v(\cdot), w(\cdot), \eta(t)) \in \Omega(T)$, let the corresponding solution $(S(t), I(t))$ of the system $(2.6)$ be defined on the maximum interval $\left[0, t_{1}\right)$, where $t_{1}$ is either a finite positive number, or $+\infty[17,24]$. Using the equality (2.3) and formulas (2.4) the third equation of the system (2.1) can be rewritten as

$$
\dot{R}(t)=-\lambda w(t)+u(t) S(t)+\eta(t) I(t) .
$$

Integration of equations of the system (2.6) leads to the inequalities:

$$
S(t)>0, \quad I(t)>0, \quad t \in\left[0, t_{1}\right) .
$$

Integrating of the equation (2.8) and then using in its subsequent analysis the equality (2.3) and formulas (2.4), we obtain the inequality

$$
R(t)>0, \quad t \in\left[0, t_{1}\right) .
$$

Then, from the relationships $(2.3),(2.10)$ we find the inequality

$$
S(t)+I(t)<N, \quad t \in\left[0, t_{1}\right) .
$$

Finally, from [17] and inequalities (2.9), (2.11) it immediately follows that the value $t_{1}$ is equal to $+\infty$, and that for given $T$ the solution $(S(t), I(t))$ of the system (2.6) corresponding to the controls $(u(\cdot), v(\cdot), w(\cdot), \eta(t)) \in \Omega(T)$, can be continued to the segment $[0, T]$ under simultaneous satisfaction of the inequalities (2.7). This completes the proof.

Remark 2.2. The inequalities (2.7) imply that the set

$$
\left\{(S, I) \in \mathrm{R}^{2}: S>0, I>0, S+I<N\right\}
$$

is an invariant set of the system (2.6).

\section{Optimal Control Problem and the Pontryagin Maximum Principle}

For the system (2.6) we consider the following optimal control problem:

$$
J(u, v, w, \eta)=\int_{0}^{T} I(t) d t \rightarrow \min _{(u(\cdot), v(\cdot), w(\cdot), \eta(\cdot)) \in \Omega(T)}
$$

for the minimization of the total level of infection on the given time interval $[0, T]$. The existence of the optimal controls $\left(u_{*}(t), v_{*}(t), w_{*}(t), \eta_{*}(t)\right)$ and the corresponding optimal trajectory $\left(S_{*}(t), I_{*}(t)\right)$, 
$t \in[0, T]$ for the problem (2.6), (3.1) follows from the restrictions (2.7) and Theorem 4 in Chapter 4 [24]. To apply the Pontryagin Maximum Principle [27], we define the Hamiltonian

$$
H\left(S, I, \psi_{1}, \psi_{2}, u, v, w, \eta\right)=-I+(\lambda w-v S I-u S) \psi_{1}+(v S I-\eta I) \psi_{2},
$$

where $\psi_{1}, \psi_{2}$ are adjoint variables. By virtue of the Pontryagin Maximum Principle there exists a nontrivial solution $\left(\psi_{1}(t), \psi_{2}(t)\right)$ of the adjoint system:

$$
\left\{\begin{array}{l}
\dot{\psi}_{1}(t)=-v_{*}(t) I_{*}(t)\left(\psi_{2}(t)-\psi_{1}(t)\right)+u_{*}(t) \psi_{1}(t), t \in[0, T] \\
\dot{\psi}_{2}(t)=-v_{*}(t) S_{*}(t)\left(\psi_{2}(t)-\psi_{1}(t)\right)+\eta_{*}(t) \psi_{2}(t)+1 \\
\psi_{1}(T)=0, \psi_{2}(T)=0
\end{array}\right.
$$

such that the controls $\left(u_{*}(t), v_{*}(t), w_{*}(t), \eta_{*}(t)\right)$ maximize the Hamiltonian

$$
H\left(S_{*}(t), I_{*}(t), \psi_{1}(t), \psi_{2}(t), u, v, w, \eta\right)
$$

with respect to values $(u, v, w, \eta)$ for almost all $t \in[0, T]$, and therefore satisfy the relationships:

$$
\begin{gathered}
u_{*}(t)=\left\{\begin{array}{lll}
u_{\max }, & \text { if } & L_{u}(t)>0, \\
\forall u \in\left[u_{\min }, u_{\max }\right], & \text { if } & L_{u}(t)=0, \\
u_{\min }, & \text { if } & L_{u}(t)<0,
\end{array}\right. \\
v_{*}(t)=\left\{\begin{array}{lll}
v_{\max }, & \text { if } & L_{v}(t)>0, \\
\forall v \in\left[v_{\min }, v_{\max }\right], & \text { if } & L_{v}(t)=0, \\
v_{\min }, & \text { if } & L_{v}(t)<0,
\end{array}\right. \\
w_{*}(t)=\left\{\begin{array}{lll}
1, & \text { if } & L_{w}(t)>0, \\
\forall w \in[0,1], & \text { if } & L_{w}(t)=0, \\
0, & \text { if } & L_{w}(t)<0,
\end{array}\right. \\
\eta_{*}(t)=\left\{\begin{array}{lll}
\eta_{\max }, & \text { if } & L_{\eta}(t)>0, \\
\forall \eta \in\left[\eta_{\min }, \eta_{\max }\right], & \text { if } & L_{\eta}(t)=0, \\
\eta_{\min }, & \text { if } & L_{\eta}(t)<0 .
\end{array}\right.
\end{gathered}
$$

Here $L_{u}(t), L_{v}(t), L_{w}(t), L_{\eta}(t)$ are the switching functions, which are related to the solution $\left(\psi_{1}(t), \psi_{2}(t)\right)$ of the system (3.3) by the equalities:

$$
L_{u}(t)=-\psi_{1}(t), \quad L_{v}(t)=\psi_{2}(t)-\psi_{1}(t), \quad L_{w}(t)=\psi_{1}(t), \quad L_{\eta}(t)=-\psi_{2}(t) .
$$

Moreover, the Hamiltonian

$$
H\left(S_{*}(t), I_{*}(t), \psi_{1}(t), \psi_{2}(t), u_{*}(t), v_{*}(t), w_{*}(t), \eta_{*}(t)\right)
$$

corresponding to the systems $(2.6),(3.3)$ is constant on the segment $[0, T]$.

The systems (2.6), (3.3) and relationships (3.4)-(3.7) form a TPBVP for the Maximum Principle. The optimal controls $\left(u_{*}(t), v_{*}(t), w_{*}(t), \eta_{*}(t)\right)$, optimal trajectory $\left(S_{*}(t), I_{*}(t)\right)$ and the corresponding solution $\left(\psi_{1}(t), \psi_{2}(t)\right)$ of the adjoint system (3.3) satisfy this problem. We will study the TPBVP for the Maximum Principle in depth.

The equalities:

$$
L_{w}(t)=-L_{u}(t), \quad L_{u}(t)=L_{v}(t)+L_{\eta}(t)
$$

follow immediately from the relationships (3.8). Hence, in order to study the behavior of the switching functions, we need only to study the functions $L_{u}(t), L_{v}(t)$, and then the behavior of the function $L_{\eta}(t)$. 
By comparison of the system (3.3) and equalities (3.8), we obtain the Cauchy problem for the switching functions $L_{u}(t)$ and $L_{v}(t)$ as

$$
\left\{\begin{array}{l}
\dot{L}_{u}(t)=u_{*}(t) L_{u}(t)+v_{*}(t) I_{*}(t) L_{v}(t), t \in[0, T] \\
\dot{L}_{v}(t)=\left(u_{*}(t)-\eta_{*}(t)\right) L_{u}(t)+\left(v_{*}(t)\left(I_{*}(t)-S_{*}(t)\right)+\eta_{*}(t)\right) L_{v}(t)+1, \\
L_{u}(T)=0, L_{v}(T)=0
\end{array}\right.
$$

and the Cauchy problem for the switching function $L_{\eta}(t)$ as

$$
\left\{\begin{array}{l}
\dot{L}_{\eta}(t)=\eta_{*}(t) L_{\eta}(t)+v_{*}(t) S_{*}(t) L_{v}(t)-1, t \in[0, T] \\
L_{\eta}(T)=0
\end{array}\right.
$$

The detailed analysis of the Cauchy problems (3.11), (3.12) is presented in the following section.

\section{Analysis of the Switching Functions}

At first, we study the behavior of the switching functions $L_{u}(t), L_{v}(t)$. Let us establish the validity of two following lemmas.

Lemma 4.1. The switching functions $L_{u}(t), L_{v}(t)$ are not equal to zero on any finite interval of the segment $[0, T]$.

Proof. At first, we consider the function $L_{u}(t)$. Suppose the contradiction. Let $L_{u}(t)=0$ hold on some interval $\Delta_{u} \subset[0, T]$. Then $\dot{L}_{u}(t)=0$ for all $t \in \Delta_{u}$. Therefore, by the first equation of the system (3.11), we find $L_{v}(t)=0$ for all $t \in \Delta_{u}$. Hence, we obtain $\dot{L}_{v}(t)=0$ for all $t \in \Delta_{u}$. The equality leads to a contradiction in the second equation of the system (3.11). Therefore, the assumption is false and the switching function $L_{u}(t)$ is not equal to zero on a finite interval of the segment $[0, T]$.

Now, we consider the function $L_{v}(t)$. Again we assume the contradiction. Let $L_{v}(t)=0$ hold on some interval $\Delta_{v} \subset[0, T]$. Then $\dot{L}_{v}(t)=0$ for all $t \in \Delta_{u}$. Therefore, the system (3.11) is rewritten as

$$
\left\{\begin{array}{l}
\dot{L}_{u}(t)=u_{*}(t) L_{u}(t), t \in \Delta_{v} \\
\left(u_{*}(t)-\eta_{*}(t)\right) L_{u}(t)+1=0
\end{array}\right.
$$

From the first equation of this system it follows that the function $L_{u}(t)$ does not vanish at any point of the interval $\Delta_{v}$. Otherwise, this function will be equal to zero everywhere on the interval which contradicts the second equation of the system (4.1). Now, for example, let $\Delta_{v}=(\alpha, \nu)$. Then, by the first equation of the system (4.1), we find

$$
L_{u}(t)=L_{0} \mathrm{e}^{-\int_{t}^{\nu} u_{*}(\xi) d \xi}, L_{0} \neq 0 ; t \in \Delta_{v} .
$$

By (3.4), the control $u_{*}(t)$ is the constant function on the interval $\Delta_{v}$ that takes a value from $\left\{u_{\min } ; u_{\max }\right\}$.

The equation from (3.12) is rewritten as

$$
\dot{L}_{\eta}(t)=\eta_{*}(t) L_{\eta}(t)-1 \text {. }
$$

Let the equality $L_{\eta}\left(t_{0}\right)=0$ hold at some time $t_{0} \in \Delta_{v}$. Then, by $(4.3)$, we have $\dot{L}_{\eta}\left(t_{0}\right)<0$. Hence, the function $L_{\eta}(t)$ changes its value from positive to negative and, by (3.7), the corresponding control $\eta_{*}(t)$ switches at the time $t_{0}$ from the value $\eta_{\max }$ to the value $\eta_{\min }$ on the interval $\Delta_{v}$. Therefore, there exists a subinterval $\Delta_{0} \subseteq \Delta_{v}$, on which the control $\eta_{*}(t)$ is a constant function taking values from $\left\{\eta_{\min } ; \eta_{\max }\right\}$. By (4.2), the second equation of the system (4.1) can be rewritten as

$$
\left(u_{*}-\eta_{*}\right) L_{0}+\mathrm{e}^{\int} \int^{\nu} u_{*}(\xi) d \xi=0, t \in \Delta_{0} .
$$


Let us analyze this relationship. If the equality $u_{*}=\eta_{*}$ holds, then the formula (4.4) is contradictory. If the inequality $\left(u_{*}-\eta_{*}\right) L_{0}>0$ is valid, then the equality (4.4) is contradictory as well. At last, if $\left(u_{*}-\eta_{*}\right) L_{0}<0$ holds, then in the relationship (4.4) the value $\left(u_{*}-\eta_{*}\right) L_{0}$ is the negative constant and the second expression is the positive decreasing function. Hence, the equality (4.4) is also contradictory. Therefore, our assumption was wrong, and the switching function $L_{v}(t)$ is not equal to zero on a finite interval of the segment $[0, T]$. This completes the proof.

Remark 4.2. By Lemma 4.1 and relationships (3.4), (3.5), the optimal controls $u_{*}(t), v_{*}(t)$ are piecewise constant functions taking values from $\left\{u_{\min } ; u_{\max }\right\},\left\{v_{\min } ; v_{\max }\right\}$, respectively.

Lemma 4.3. The switching function $L_{u}(t)$ takes only positive values for all $t \in[0, T)$.

Proof. Using the substitutions:

$$
\phi_{1}(t)=L_{u}(t), \quad \chi_{1}(t)=L_{v}(t)+h(t) L_{u}(t),
$$

where $h(t)$ is a function to be determined, transform the matrix of the system (3.11) to the upper triangular form:

$$
\left(\begin{array}{cc}
u_{*}(t) & v_{*}(t) I_{*}(t) \\
\left(u_{*}(t)-\eta_{*}(t)\right) & \left(v_{*}(t)\left(I_{*}(t)-S_{*}(t)\right)+\eta_{*}(t)\right)
\end{array}\right) \rightarrow\left(\begin{array}{c}
* * \\
0 *
\end{array}\right) .
$$

Functions $\phi_{1}(t)$ and $\chi_{1}(t)$ satisfy the system of differential equations:

$$
\left\{\begin{aligned}
\dot{\phi}_{1}(t)= & \left(u_{*}(t)-v_{*}(t) I_{*}(t) h(t)\right) \phi_{1}(t)+v_{*}(t) I_{*}(t) \chi_{1}(t) \\
\dot{\chi}_{1}(t)= & {\left[\dot{h}(t)-v_{*}(t) I_{*}(t) h^{2}(t)-\left(v_{*}(t)\left(I_{*}(t)-S_{*}(t)\right)+\left(\eta_{*}(t)-u_{*}(t)\right)\right) h(t)\right.} \\
& \left.-\left(\eta_{*}(t)-u_{*}(t)\right)\right] \phi_{1}(t) \\
& +\left(v_{*}(t)\left(I_{*}(t)-S_{*}(t)\right)+\eta_{*}(t)+v_{*}(t) I_{*}(t) h(t)\right) \chi_{1}(t)+1 .
\end{aligned}\right.
$$

Let choose the function $h(t)$ to make the expression inside the square brackets equal to zero. Then this function satisfies the Riccati equation

$$
\dot{h}(t)=v_{*}(t) I_{*}(t) h^{2}(t)+\left(v_{*}(t)\left(I_{*}(t)-S_{*}(t)\right)+\left(\eta_{*}(t)-u_{*}(t)\right)\right) h(t)+\left(\eta_{*}(t)-u_{*}(t)\right) .
$$

Introduce the functions:

$$
a_{1}(t)=v_{*}(t) I_{*}(t), \quad b_{1}(t)=v_{*}(t)\left(I_{*}(t)-S_{*}(t)\right)+\left(\eta_{*}(t)-u_{*}(t)\right), \quad c_{1}(t)=\eta_{*}(t)-u_{*}(t),
$$

then by (4.6), we obtain the non-autonomous differential Riccati equation

$$
\dot{h}(t)=a_{1}(t) h^{2}(t)+b_{1}(t) h(t)+c_{1}(t) .
$$

Lemma 2.1 leads to the following estimations for the functions $a_{1}(t), b_{1}(t), c_{1}(t)$ :

$$
\begin{aligned}
\left|a_{1}(t)\right|= & a_{1}(t) \leq v_{\max } N=A_{1}, \\
\left|b_{1}(t)\right|= & \sqrt{\left(v_{*}(t)\left(I_{*}(t)-S_{*}(t)\right)+\left(\eta_{*}(t)-u_{*}(t)\right)\right)^{2}} \\
& \leq \sqrt{2 v_{*}^{2}(t)\left(I_{*}(t)-S_{*}(t)\right)^{2}+2\left(\eta_{*}(t)-u_{*}(t)\right)^{2}} \\
& \leq \sqrt{2 v_{*}^{2}(t) I_{*}^{2}(t)+2 v_{*}^{2}(t) S_{*}^{2}(t)+2 \eta_{*}^{2}(t)+2 u_{*}^{2}(t)} \\
& \leq \sqrt{4 v_{\max }^{2} N^{2}+2 \eta_{\max }^{2}+2 u_{\max }^{2}}=B_{1} \\
\left|c_{1}(t)\right|= & \sqrt{\left(\eta_{*}(t)-u_{*}(t)\right)^{2}} \leq \sqrt{\eta_{*}^{2}(t)+u_{*}^{2}(t)} \leq \sqrt{\eta_{\max }^{2}+u_{\max }^{2}}=C_{1} .
\end{aligned}
$$


Here, the values of $A_{1}, B_{1}, C_{1}$ satisfy the following chain of relationships:

$$
\begin{gathered}
B_{1}^{2}-4 A_{1} C_{1}=4 v_{\max }^{2} N^{2}+2 \eta_{\max }^{2}+2 u_{\max }^{2}-4 v_{\max } N \sqrt{\eta_{\max }^{2}+u_{\max }^{2}} \\
=2 v_{\max }^{2} N^{2}+2\left(v_{\max } N-\sqrt{\eta_{\max }^{2}+u_{\max }^{2}}\right)^{2}>0 .
\end{gathered}
$$

Now, we will show that for the equation (4.7) there exists a solution defined on the entire segment $[0, T]$. Assume the contradiction, i. e. that an arbitrary solution $h(t)$ of the equation (4.7) is defined on the interval $\left[0, t_{1}\right), t_{1} \in(0, T]$, which is the biggest interval of the existence of this solution. Then, from Lemma in $\S 14$ of Chapter 4 [9] for solution $h(t)$ it follows the relationship

$$
\lim _{t \rightarrow t_{1}-0}|h(t)|=+\infty \text {. }
$$

Its validity leads to the existence of a number $\rho>0$ and a value $t_{0} \in\left[0, t_{1}\right)$, for which the inequality $|h(t)| \geq \rho$ holds for all $t \in\left[t_{0}, t_{1}\right)$. Here the values $\rho$ and $t_{0}$ will be defined below.

Let us evaluate on the interval $\left[t_{0}, t_{1}\right)$ the derivative of the function $|h(t)|$ regarding the equation (4.7). We have the equality

$$
\frac{d}{d t}(|h(t)|)=|h(t)|^{-1} \cdot\left[a_{1}(t) h^{3}(t)+b_{1}(t) h^{2}(t)+c_{1}(t) h(t)\right] .
$$

Next, using inequalities (4.8), we will estimate the upper boundary of the expression inside square brackets:

$$
\begin{aligned}
a_{1}(t) h^{3}(t)+b_{1}(t) h^{2}(t) & +c_{1}(t) h(t) \leq\left|a_{1}(t)\right||h(t)|^{3}+\left|b_{1}(t)\right||h(t)|^{2}+\left|c_{1}(t)\right||h(t)| \\
& \leq A_{1}|h(t)|^{3}+B_{1}|h(t)|^{2}+C_{1}|h(t)| .
\end{aligned}
$$

Substituting these inequalities into the formula (4.11) we find a differential inequality

$$
\frac{d}{d t}(|h(t)|) \leq A_{1}|h(t)|^{2}+B_{1}|h(t)|+C_{1}, \quad t \in\left[t_{0}, t_{1}\right) .
$$

Now, we will consider the quadratic equation

$$
A_{1} K^{2}-B_{1} K+C_{1}=0
$$

By (4.9), it is easy to see that its discriminant is positive.

Now, for all values $h$ that $|h| \geq \rho$ we will introduce the function $V(h)=|h|+K_{0}$, where a value $K_{0}$ is a biggest root of the equation (4.13), i. e.

$$
K_{0}=\frac{B_{1}+\sqrt{B_{1}^{2}-4 A_{1} C_{1}}}{2 A_{1}} .
$$

Let us rewrite the differential inequality (4.12) for the function $V(h)$. We have the inequality

$$
\frac{d}{d t}(V(h(t))) \leq A_{1}\left(V(h(t))-K_{0}\right)^{2}+B_{1}\left(V(h(t))-K_{0}\right)+C_{1}
$$

After necessary transformations regarding relationship $A_{1} K_{0}^{2}-B_{1} K_{0}+C_{1}=0$, we find the differential inequality

$$
\frac{d}{d t}(V(h(t))) \leq A_{1} V^{2}(h(t))-\left(2 A_{1} K_{0}-B_{1}\right) V(h(t)), \quad t \in\left[t_{0}, t_{1}\right) .
$$

At last, let us consider the auxiliary Cauchy problem

$$
\left\{\begin{array}{l}
\dot{q}(t)=A_{1} q^{2}(t)-\left(2 A_{1} K_{0}-B_{1}\right) q(t), t \in\left[t_{0}, t_{1}\right], \\
q\left(t_{0}\right)=q_{0}, q_{0} \geq K_{0}+\rho .
\end{array}\right.
$$


Here the value $q_{0}$ satisfy the inequality

$$
q_{0}>\frac{2 A_{1} K_{0}-B_{1}}{A_{1}}
$$

Solving the corresponding Bernoulli equation and satisfying the initial condition, we find the solution to (4.15) as

$$
q(t)=\left(\frac{A_{1}}{2 A_{1} K_{0}-B_{1}}+\left[\frac{1}{q_{0}}-\frac{A_{1}}{2 A_{1} K_{0}-B_{1}}\right] e^{\left(2 A_{1} K_{0}-B_{1}\right)\left(t-t_{0}\right)}\right)^{-1}, t \in\left[t_{0}, t_{1}\right] .
$$

In this equality, we assume that the values of $\rho$ and $t_{0}$ are such that the expression in brackets is defined for all $t \in\left[t_{0}, t_{1}\right]$. We can do this, for example, by choosing for a given $\rho$, a value $t_{0}$ such that the difference $\left(t_{1}-t_{0}\right)$ is sufficiently small. By (4.16), the sum inside square brackets in (4.17) is negative, and hence $q(t)$ is a finite positive function monotonically increasing on the segment $\left[t_{0}, t_{1}\right]$. Therefore, $q(t)<q\left(t_{1}\right)$ for all $t \in\left[t_{0}, t_{1}\right)$. Hence, by the differential inequality (4.14), the Cauchy problem (4.15), Chaplygin's Theorem (Theorem 1.1 in [29]), and under the condition

$$
q_{0}=V\left(h\left(t_{0}\right)\right)=K_{0}+\left|h\left(t_{0}\right)\right|,
$$

we have the inequalities:

$$
|h(t)|<q(t)-K_{0}<q\left(t_{1}\right)-K_{0}, t \in\left(t_{0}, t_{1}\right),
$$

which contradict to (4.10). Our assumption was wrong. Therefore, there exists the solution $h(t)$ of the Riccati equation (4.7) or (4.6) that is defined on the entire segment $[0, T]$. Then the system (4.5) is also defined on this segment and can be rewritten as

$$
\left\{\begin{array}{l}
\dot{\phi}_{1}(t)=\left(u_{*}(t)-v_{*}(t) I_{*}(t) h(t)\right) \phi_{1}(t)+v_{*}(t) I_{*}(t) \chi_{1}(t) \\
\dot{\chi}_{1}(t)=\left(v_{*}(t)\left(I_{*}(t)-S_{*}(t)\right)+\eta_{*}(t)+v_{*}(t) I_{*}(t) h(t)\right) \chi_{1}(t)+1 .
\end{array}\right.
$$

The initial conditions for the functions $L_{u}(t), L_{v}(t)$ from (3.11) lead to the similar initial conditions for the functions $\phi_{1}(t), \chi_{1}(t)$ :

$$
\phi_{1}(T)=0, \quad \chi_{1}(T)=0 .
$$

At first, integrating the second equation of the system (4.18) with the corresponding initial condition from (4.19), we obtain the formula

$$
\chi_{1}(t)=-\int_{t}^{T} \mathrm{e}^{-\int_{t}^{\tau}\left(v_{*}(\xi)\left(I_{*}(\xi)-S_{*}(\xi)\right)+\eta_{*}(\xi)+v_{*}(\xi) I_{*}(\xi) h(\xi)\right) d \xi} d \tau,
$$

from which the inequality $\chi_{1}(t)<0$ follows for all $t \in[0, T)$. Then the integration of the first equation of the system (4.18) with the corresponding initial condition from (4.19) leads to the formula

$$
\phi_{1}(t)=-\int_{t}^{T} \mathrm{e}^{-\int_{t}^{\tau}\left(u_{*}(\xi)-v_{*}(\xi) I_{*}(\xi) h(\xi)\right) d \xi} v_{*}(\tau) I_{*}(\tau) \chi_{1}(\tau) d \tau,
$$

that implies the validity of the inequality $\phi_{1}(t)=L_{u}(t)>0$ for all $t \in[0, T)$. This completes the proof.

Remark 4.4. Similar arguments were used in $[13,15]$ for the analysis of attainable sets for threedimensional control nonlinear systems of differential equations, which describe the different biochemical processes. Moreover, the other approach for an extension of solutions of non-autonomous differential Riccati equations of the type (4.7) based on the non-oscillation theory was presented in [12]. 
Corollary 4.5. Lemma 4.3 and the first equality from (3.10) imply the inequality

$$
L_{w}(t)<0, \quad t \in[0, T) .
$$

Remark 4.6. By Lemma 4.3, Corollary 4.5 and relationships (3.4), (3.6), the optimal controls $u_{*}(t)$, $w_{*}(t)$ have the following types:

$$
u_{*}(t)=u_{\max }, \quad w_{*}(t)=0, \quad t \in[0, T] .
$$

Now, we study the behavior of the switching function $L_{\eta}(t)$. Let us establish the validity of three following lemmas.

Lemma 4.7. The switching function $L_{\eta}(t)$ is not equal to zero on any finite interval of the segment $[0, T]$.

Proof. Using the switching functions $L_{u}(t), L_{v}(t), L_{w}(t), L_{\eta}(t)$ rewrite the Hamiltonian (3.2), (3.9) as

$$
\begin{gathered}
H\left(S_{*}(t), I_{*}(t), \psi_{1}(t), \psi_{2}(t), u_{*}(t), v_{*}(t), w_{*}(t), \eta_{*}(t)\right) \\
=\lambda w_{*}(t) L_{w}(t)+u_{*}(t) S_{*}(t) L_{u}(t)+\eta_{*}(t) I_{*}(t) L_{\eta}(t)+v_{*}(t) S_{*}(t) I_{*}(t) L_{v}(t)-I_{*}(t) .
\end{gathered}
$$

Relationships (4.20), a constant value of the Hamiltonian (3.9) on the segment $[0, T]$ and initial conditions from (3.11), (3.12) imply that the formula (4.21) can be rewritten as

$$
u_{\max } S_{*}(t) L_{u}(t)+\eta_{*}(t) I_{*}(t) L_{\eta}(t)+v_{*}(t) S_{*}(t) I_{*}(t) L_{v}(t)-I_{*}(t)=-I_{*}(T), \quad t \in[0, T) .
$$

Now, suppose that there exists the interval $\Delta_{\eta} \subset[0, T]$, on which the equality

$$
L_{\eta}(t)=0
$$

holds. Then the second formula from (3.10) imply the equality

$$
L_{u}(t)=L_{v}(t), \quad t \in \Delta_{\eta} .
$$

Also, by (4.23), it is valid the equality $\dot{L}_{\eta}(t)=0$, which leads the equation from (3.12) to the formula

$$
v_{*}(t) S_{*}(t) L_{v}(t)=1, \quad t \in \Delta_{\eta} .
$$

From this equality and by (3.5) we conclude that $v_{*}(t)=v_{\max }$ for all $t \in \Delta_{\eta}$. Moreover, the relationship (4.25) can be rewritten as

$$
L_{v}(t)=v_{\max }^{-1} S_{*}^{-1}(t), \quad t \in \Delta_{\eta} .
$$

Substituting equalities (4.23), (4.24) and (4.26) into the formula (4.22), we obtain the contradictory chain of relationships:

$$
0<u_{\max } v_{\max }^{-1}=S_{*}(t)\left(u_{\max }+v_{\max } I_{*}(t)\right) v_{\max }^{-1} S_{*}^{-1}(t)-I_{*}(t)=-I_{*}(T)<0, t \in \Delta_{\eta} .
$$

Therefore, our assumption was wrong, and the switching function $L_{\eta}(t)$ is not equal to zero on any finite interval of the segment $[0, T]$. This completes the proof.

Remark 4.8. By Lemma 4.7 and relationship (3.7), the optimal control $\eta_{*}(t)$ is a piecewise constant function taking values from $\left\{\eta_{\min } ; \eta_{\max }\right\}$.

Lemma 4.9. There is no the value $t_{0} \in[0, T)$, for which the relationships:

$$
L_{\eta}\left(t_{0}\right)=0, \quad \dot{L}_{\eta}\left(t_{0}\right) \geq 0
$$

simultaneously hold. 
Proof. Assume the contradiction. Let there necessary exists the time $t_{0} \in[0, T)$ such that the relationships (4.27) hold. Then by (3.10) and the equation of (3.12), we obtain the relationships:

$$
L_{u}\left(t_{0}\right)=L_{v}\left(t_{0}\right), \quad L_{v}\left(t_{0}\right) \geq v_{*}^{-1}\left(t_{0}\right) S_{*}^{-1}\left(t_{0}\right) .
$$

Substituting (4.27), (4.28) into the formula (4.22) we find the contradictory chain of relationships:

$$
\begin{aligned}
0 & >-I_{*}(T)=u_{\max } S_{*}\left(t_{0}\right) L_{u}\left(t_{0}\right)+v_{*}\left(t_{0}\right) S_{*}\left(t_{0}\right) I_{*}\left(t_{0}\right) L_{v}\left(t_{0}\right)-I_{*}\left(t_{0}\right) \\
& \geq S_{*}\left(t_{0}\right)\left(u_{\max }+v_{*}\left(t_{0}\right) I_{*}\left(t_{0}\right)\right) v_{*}^{-1}\left(t_{0}\right) S_{*}^{-1}\left(t_{0}\right)-I_{*}\left(t_{0}\right)=u_{\max } v_{*}^{-1}\left(t_{0}\right)>0 .
\end{aligned}
$$

Therefore, our assumption was wrong. This completes the proof.

As a consequence of Lemma 4.9 we have the following statement.

Lemma 4.10. The switching function $L_{\eta}(t)$ takes only positive values for all $t \in[0, T)$.

Proof. From the system (3.11) it follows the equalities:

$$
L_{v}(T)=0, \quad \dot{L}_{v}(T)=1,
$$

and also the continuity of the derivative $\dot{L}_{v}(t)$ of the function $L_{v}(t)$ on a small interval adjoining to $T$. Then, the inequality $L_{v}(t)<0$ holds for all $t$ from this interval. Therefore, integrating the system (3.12) on this interval we obtain the formula

$$
L_{\eta}(t)=-\int_{t}^{T} \mathrm{e}^{-\int_{t}^{\tau} \eta_{*}(\xi) d \xi}\left(v_{*}(\tau) S_{*}(\tau) L_{v}(\tau)-1\right) d \tau
$$

from which we find the inequality $L_{\eta}(t)>0$. Then, the desired result follows from these arguments and Lemma 4.9. This completes the proof.

Remark 4.11. By Lemma 4.10 and relationship (3.7), the optimal control $\eta_{*}(t)$ has the type

$$
\eta_{*}(t)=\eta_{\max }, \quad t \in[0, T] .
$$

At last, we study the behavior of the switching function $L_{v}(t)$. For this, we rewrite the Cauchy problem (3.11), substituting into it the founded optimal controls $u_{*}(t), \eta_{*}(t)$ as

$$
\left\{\begin{array}{l}
\dot{L}_{u}(t)=u_{\max } L_{u}(t)+v_{*}(t) I_{*}(t) L_{v}(t), t \in[0, T] \\
\dot{L}_{v}(t)=\left(u_{\max }-\eta_{\max }\right) L_{u}(t)+\left(v_{*}(t)\left(I_{*}(t)-S_{*}(t)\right)+\eta_{\max }\right) L_{v}(t)+1 \\
L_{u}(T)=0, L_{v}(T)=0
\end{array}\right.
$$

The analysis of the Cauchy problem (4.30) reveals that depending on the value of $\left(u_{\max }-\eta_{\max }\right)$, there are two distinctive cases, namely $u_{\max }-\eta_{\max } \geq 0$ and $u_{\max }-\eta_{\max }<0$, which should be investigated separately.

Case 4.12. Let $u_{\max }-\eta_{\max } \geq 0$. In the proof of Lemma 4.10 it was shown the existence of the interval adjoining to $T$, on which the inequality $L_{v}(t)<0$ holds. Let us show that this inequality is true for all $t \in[0, T)$. Suppose the contradiction. Then, there necessary exists the time $t_{0} \in[0, T)$ such that:

$$
\begin{gathered}
L_{v}\left(t_{0}\right)=0, \\
L_{v}(t)<0, \quad t \in\left(t_{0}, T\right), \\
\dot{L}_{v}\left(t_{0}\right) \leq 0 .
\end{gathered}
$$

By Lemma 4.3, the equality (4.31) and the second equation of the system (4.30), it is valid the inequality $\dot{L}_{v}\left(t_{0}\right)>0$, which contradicts to (4.32). Therefore, our assumption was wrong, and we have the inequality

$$
L_{v}(t)<0, \quad t \in[0, T) .
$$


Remark 4.13. By relationships (3.5), (4.33), in Case 4.12 the optimal control $v_{*}(t)$ has the type

$$
v_{*}(t)=v_{\min }, \quad t \in[0, T] .
$$

Case 4.14. Let $u_{\max }-\eta_{\max }<0$. Introduce the auxiliary function $Q(t)=\left(u_{\max }-\eta_{\max }\right) L_{u}(t)+1$, and then obtain the Cauchy problem for the functions $L_{v}(t), Q(t)$ as

$$
\left\{\begin{array}{l}
\dot{L}_{v}(t)=\left(v_{*}(t)\left(I_{*}(t)-S_{*}(t)\right)+\eta_{\max }\right) L_{v}(t)+Q(t), t \in[0, T] \\
\dot{Q}(t)=\left(u_{\max }-\eta_{\max }\right) v_{*}(t) I_{*}(t) L_{v}(t)+u_{\max } Q(t)-\left(u_{\max }-\eta_{\max }\right), \\
L_{v}(T)=0, Q(T)=1
\end{array}\right.
$$

Now, using the substitutions:

$$
\phi_{2}(t)=L_{v}(t), \chi_{2}(t)=Q(t)+g(t) L_{v}(t),
$$

where $g(t)$ is a function to be determined, transform the matrix of the system (4.35) to the upper triangular form:

$$
\left(\begin{array}{cc}
\left(v_{*}(t)\left(I_{*}(t)-S_{*}(t)\right)+\eta_{\max }\right) & 1 \\
\left(u_{\max }-\eta_{\max }\right) v_{*}(t) I_{*}(t) & u_{\max }
\end{array}\right) \rightarrow\left(\begin{array}{c}
* * \\
0 *
\end{array}\right) .
$$

Functions $\phi_{2}(t)$ and $\chi_{2}(t)$ satisfy the system of differential equations:

$$
\left\{\begin{aligned}
\dot{\phi}_{2}(t)= & \left(v_{*}(t)\left(I_{*}(t)-S_{*}(t)\right)+\eta_{\max }-g(t)\right) \phi_{2}(t)+\chi_{2}(t) \\
\dot{\chi}_{2}(t)= & {\left[\dot{g}(t)-g^{2}(t)+\left(v_{*}(t)\left(I_{*}(t)-S_{*}(t)\right)+\left(\eta_{\max }-u_{\max }\right)\right) g(t)\right.} \\
& \left.-\left(\eta_{\max }-u_{\max }\right) v_{*}(t) I_{*}(t)\right] \phi_{2}(t)+\left(g(t)+u_{\max }\right) \chi_{2}(t)+\left(\eta_{\max }-u_{\max }\right) .
\end{aligned}\right.
$$

Let choose the function $g(t)$ to make the expression inside the square brackets equal to zero. Then this function satisfies the Riccati equation

$$
\dot{g}(t)=g^{2}(t)-\left(v_{*}(t)\left(I_{*}(t)-S_{*}(t)\right)+\left(\eta_{\max }-u_{\max }\right)\right) g(t)+\left(\eta_{\max }-u_{\max }\right) v_{*}(t) I_{*}(t) .
$$

Introduce the functions:

$$
a_{2}(t)=1, \quad b_{2}(t)=v_{*}(t)\left(I_{*}(t)-S_{*}(t)\right)+\left(\eta_{\max }-u_{\max }\right), \quad c_{2}(t)=\left(\eta_{\max }-u_{\max }\right) v_{*}(t) I_{*}(t),
$$

then by (4.37), we obtain the non-autonomous differential Riccati equation

$$
\dot{g}(t)=a_{2}(t) g^{2}(t)+b_{2}(t) g(t)+c_{2}(t) .
$$

Lemma 2.1 leads to the following estimations for the functions $a_{2}(t), b_{2}(t), c_{2}(t)$ :

$$
\begin{aligned}
\left|a_{2}(t)\right|= & a_{2}(t)=1=A_{2}, \\
\left|b_{2}(t)\right|= & \sqrt{\left(v_{*}(t)\left(I_{*}(t)-S_{*}(t)\right)+\left(\eta_{\max }-u_{\max }\right)\right)^{2}} \\
& \leq \sqrt{2 v_{*}^{2}(t)\left(I_{*}(t)-S_{*}(t)\right)^{2}+2\left(\eta_{\max }-u_{\max }\right)^{2}} \\
& \leq \sqrt{2 v_{*}^{2}(t) I_{*}^{2}(t)+2 v_{*}^{2}(t) S_{*}^{2}(t)+2\left(\eta_{\max }-u_{\max }\right)^{2}} \\
& \leq \sqrt{4 v_{\max }^{2} N^{2}+2\left(\eta_{\max }-u_{\max }\right)^{2}}=B_{2}, \\
\left|c_{2}(t)\right|= & c_{2}(t) \leq\left(\eta_{\max }-u_{\max }\right) v_{\max } N=C_{2} .
\end{aligned}
$$

Here, the values of $A_{2}, B_{2}, C_{2}$ satisfy the following chain of relationships:

$$
\begin{gathered}
B_{2}^{2}-4 A_{2} C_{2}=4 v_{\max }^{2} N^{2}+2\left(\eta_{\max }-u_{\max }\right)^{2}-4 v_{\max } N\left(\eta_{\max }-u_{\max }\right) \\
=2 v_{\max }^{2} N^{2}+2\left(v_{\max } N-\left(\eta_{\max }-u_{\max }\right)\right)^{2}>0 .
\end{gathered}
$$


Further, using relationships (4.39), (4.40) we conduct arguments that are similar to these that were applied in Lemma 4.3. In result, we obtain the solution $g(t)$ of the Riccati equation (4.38) or (4.37) that is defined on the entire segment $[0, T]$. Then the system (4.36) is also defined on this segment and can be rewritten as

$$
\left\{\begin{array}{l}
\dot{\phi}_{2}(t)=\left(v_{*}(t)\left(I_{*}(t)-S_{*}(t)\right)+\eta_{\max }-g(t)\right) \phi_{2}(t)+\chi_{2}(t), \\
\dot{\chi}_{2}(t)=\left(g(t)+u_{\max }\right) \chi_{2}(t)+\left(\eta_{\max }-u_{\max }\right) .
\end{array}\right.
$$

The initial conditions for the functions $L_{v}(t), Q(t)$ from (4.35) lead to the similar initial conditions for the functions $\phi_{2}(t), \chi_{2}(t)$ :

$$
\phi_{2}(T)=0, \quad \chi_{2}(T)=1 .
$$

From the second equation of the system (4.41) it follows that, if there exists a moment of time $\tau \in[0, T)$ such that $\chi_{2}(\tau)=0$, then $\dot{\chi}_{2}(\tau)>0$ holds. From here and the second formula of (4.42) we conclude that the function $\chi_{2}(t)$ has at most one zero on the interval $[0, T)$ and, if this zero occurs, then $\chi_{2}(t)$ changes its value from negative to positive. Then, by the generalized Rolle's Theorem [10] applied to the first equation of the system (4.41) and the first formula of (4.42), the function $\phi_{2}(t)=L_{v}(t)$ has at most one zero on the interval $[0, T)$. Hence, from the system (4.35) we obtain for the switching function $L_{v}(t)$ the formula

$$
L_{v}(t)\left\{\begin{array}{l}
>0, \text { if } \quad 0 \leq t<\theta_{*}, \\
=0, \text { if } \quad t=\theta_{*}, \\
<0, \text { if } \quad \theta_{*}<t \leq T,
\end{array}\right.
$$

where $\theta_{*} \in[0, T)$ is the zero of the function $L_{v}(t)$.

Remark 4.15. By relationships (3.5), (4.43), in Case 4.14 the optimal control $v_{*}(t)$ has the type

$$
v_{*}(t)=\left\{\begin{array}{lll}
v_{\max }, & \text { if } & 0 \leq t<\theta_{*}, \\
v_{\min }, & \text { if } & \theta_{*} \leq t \leq T,
\end{array}\right.
$$

where $\theta_{*} \in[0, T)$ is the moment of switching.

From formulas (4.20), (4.29), (4.34), (4.44) it follows the conclusions:

- In Case 4.12, by Theorem 7.1.1 [5], the optimal controls $\left(u_{*}(t), v_{*}(t), w_{*}(t), \eta_{*}(t)\right)$ are unique, and hence provide the global minimum of the objective functional (3.1). Moreover, these controls together with the optimal trajectory $\left(S_{*}(t), I_{*}(t)\right)$ and the corresponding solution $\left(\psi_{1}(t), \psi_{2}(t)\right)$ of the adjoint system (3.3) are the unique solution of the TPBVP for the Maximum Principle.

- In Case 4.14 we note that the TPBVP for the Maximum Principle besides the optimal solution

$$
\left\{\left(u_{*}(t), v_{*}(t), w_{*}(t), \eta_{*}(t)\right),\left(S_{*}(t), I_{*}(t)\right),\left(\psi_{1}(t), \psi_{2}(t)\right)\right\}
$$

can also have other solutions

$$
\left\{(\widetilde{u}(t), \widetilde{v}(t), \widetilde{w}(t), \widetilde{\eta}(t)),(\widetilde{S}(t), \widetilde{I}(t)),\left(\varphi_{1}(t), \varphi_{2}(t)\right)\right\},
$$

which are called extremals solutions; where $(\widetilde{u}(t), \widetilde{v}(t), \widetilde{w}(t), \widetilde{\eta}(t))$ are the extremal controls, $(\widetilde{S}(t), \widetilde{I}(t))$ is the extremal trajectory and $\left(\varphi_{1}(t), \varphi_{2}(t)\right)$ is the corresponding solution of the adjoint system (3.3). From the arguments above it is easy to see that the extremal controls $(\widetilde{u}(t), \widetilde{v}(t), \widetilde{w}(t), \widetilde{\eta}(t))$ have the types similar to (4.20), (4.29), (4.34), (4.44). In [11,20,21] it was shown that the TPBVP for the Maximum Principle has unique solution for a small value of $T$. Therefore, as in Case 4.12, the optimal controls $\left(u_{*}(t), v_{*}(t), w_{*}(t), \eta_{*}(t)\right)$ provide the global minimum of the objective functional (3.1). In general, this is not the case. Hence, finding the global minimum of the objective functional (3.1) corresponding to the optimal solution of the problem (2.6), (3.1) is possible only numerically. Detailed description of the corresponding numerical algorithm is given in the following section. 
Using formulas (2.4) we can return from controls $(u(t), v(t), w(t), \eta(t))$ to the original controls $(p(t), q(t), r(t), s(t))$. Relationships (4.20), (4.29), (4.34), (4.44) show that for all values $q_{\max }$ and $\left(\gamma+s_{\max }\right)$, corresponding to $u_{\max }$ and $\eta_{\max }$, the optimal controls $p_{*}(t), q_{*}(t), s_{*}(t)$ are constant and take the maximum values:

$$
p_{*}(t)=1, \quad q_{*}(t)=q_{\max }, \quad s_{*}(t)=s_{\max }, \quad t \in[0, T] .
$$

The type of the optimal control $r_{*}(t)$ depends on $q_{\max }$ and $\left(\gamma+s_{\max }\right)$. Specifically, if $q_{\max } \geq \gamma+s_{\max }$, then the control $r_{*}(t)$ is also constant and takes the maximum value:

$$
r_{*}(t)=r_{\max }, \quad t \in[0, T] .
$$

If $q_{\max }<\gamma+s_{\max }$, then the control $r_{*}(t)$ is the piecewise constant function with at most one switching of the type:

$$
r_{*}(t)=\left\{\begin{array}{lll}
r_{\min }, & \text { if } \quad 0 \leq t<\theta_{*} \\
r_{\max }, & \text { if } \quad \theta_{*} \leq t \leq T
\end{array}\right.
$$

where $\theta_{*} \in[0, T)$ is the moment of switching.

\section{Numerical Solution of the Optimal Control Problem}

Solution to the optimal control problem (2.6), (3.1) for $u_{\max }<\eta_{\max }$ is conducted numerically using the following arguments. For an arbitrary value $\theta \in[0, T]$ we define control $v_{\theta}(t)$ as

$$
v_{\theta}(t)= \begin{cases}v_{\max }, & \text { if } \quad 0 \leq t \leq \theta \\ v_{\min }, & \text { if } \quad \theta<t \leq T\end{cases}
$$

It is easy to see that the control $v_{\theta}(t)$ defined in this way includes all possible types $(4.34),(4.44)$ of the optimal control $v_{*}(t)$. Additionally, we use formulas (4.20), (4.29) for the optimal controls $u_{*}(t), w_{*}(t)$, $\eta_{*}(t)$.

System (2.6) can be integrated on interval $[0, T]$ with controls:

$$
u(t)=u_{\max }, v(t)=v_{\theta}(t), w(t)=0, \quad \eta(t)=\eta_{\max }, \quad t \in[0, T] .
$$

The function $I_{\theta}(t)$, which with the function $S_{\theta}(t)$ correspond to these controls, then should be substituted into the functional (3.1). This yields a function

$$
F(\theta)=J\left(u_{\max }, v_{\theta}, 0, \eta_{\max }\right), \quad \theta \in[0, T],
$$

which is a function of a single variable $\theta \in[0, T]$, and hence the problem (2.6), (3.1) is now reduced to a constrained minimization problem

$$
F(\theta) \rightarrow \min _{\theta \in[0, T]} .
$$

This minimization problem is solved numerically. In order to find the global minimum of the function $F(\theta)$, the overlapping method combined with the iterative gradient projection method are used [30]. The corresponding numerical algorithm is written in MAPLE. Since the Pontryagin Maximum Principle is only a necessary optimality condition, then the function $F(\theta)$ can have local minima different from the global minimum. To take this into account, we introduce a grid of points on the interval $[0, T]$. The partitioning of the grid depends on the Lipschitz constant of the function $F(\theta)$. We select the points of this grid as initial iterations for the following multi-used gradient projection method, and find approximately local minima of the function $F(\theta)$. Finally, comparing the obtained results we approximately determine the global minimum, which is the solution of the original problem. The value of $\theta_{*}$ corresponding to this minimum is the desired moment of switching of the optimal control $v_{*}(t)$. 
Remark 5.1. Similar approach for the numerical solution of the optimal control problems for the model of waste water biological treatment was considered in [14].

As we obtained analytically, depending on the parameters of the model and the difference between $u_{\max }$ and $\eta_{\max }$, given by Case 4.12 or Case 4.14, two different optimal strategies can occur. Thus, in Case 4.12 , if $u_{\max } \geq \eta_{\max }$, then one must take maximum effort (all four controls must take maximum values) over the time interval $[0, T]$. In turn, in Case 4.14, if $u_{\max }<\eta_{\max }$, the optimal control $v_{*}(t)$ can switch at the time $\theta_{*} \in[0, T)$ from the value $v_{\max }$ to the value $v_{\min }$. Hence, the optimal control $r_{*}(t)$ would switch at the same moment $\theta_{*}$ from the value $r_{\min }$ to the value $r_{\max }$. In order to illustrate Case 4.12, we conducted numerical calculations using the following values of the initial conditions $S_{0}, I_{0}$ and parameters $\lambda, T, u_{\max }, v_{\min }, v_{\max }$ and $\eta_{\max }$ of the system (2.6):

$$
\begin{aligned}
& S_{0}=50.0, \quad I_{0}=1.0, \lambda=10.0, T=10.0, \\
& u_{\max }=1.2, \quad v_{\min }=0.1, \quad v_{\max }=0.3, \quad \eta_{\max }=0.7 .
\end{aligned}
$$

In order to illustrate Case 4.14, we conducted numerical calculations using the following values of $S_{0}, I_{0}$, $\lambda, T, u_{\max }, v_{\min }, v_{\max }, \eta_{\max }$ for the system (2.6):

$$
\begin{aligned}
& S_{0}=50.0, \quad I_{0}=1.0, \quad \lambda=10.0, T=10.0, \\
& u_{\max }=1.2, \quad v_{\min }=0.1, \quad v_{\max }=4.0, \quad \eta_{\max }=4.0 .
\end{aligned}
$$

Values of $u_{\min }, \eta_{\min }$ are not specified, since they do not influence the optimal solution of the original problem.

Figures 1, 2 show the graphs of the optimal solutions $S_{*}(t), I_{*}(t)$ and correspond to Case 4.12 and Case 4.14, respectively. In both Figures, the optimal solution $S_{*}(t)$ is shown as a green curve, and the solution $I_{*}(t)$ is shown in blue. In Figure 2 the optimal solutions are obtained for the piecewise constant control $v_{*}(t)$ that switches at the time $\theta_{*}=0.75$ from the value $v_{\max }$ to the value $v_{\min }$.

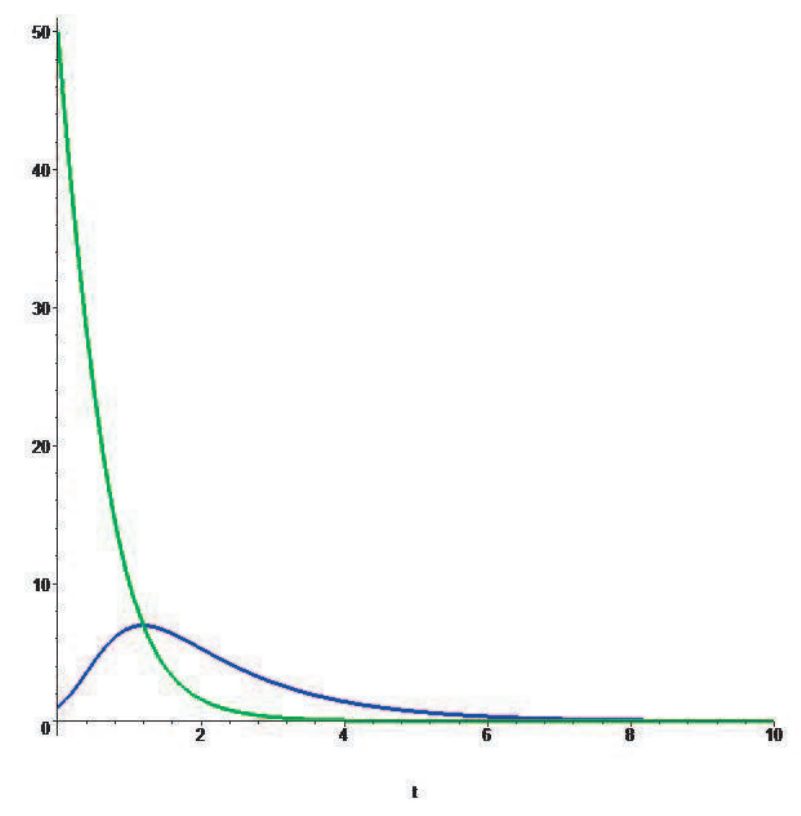

Figure 1. Case 4.12. Graphs of the optimal solutions $S_{*}(t), I_{*}(t)$ for $v_{\max }=0.3, \eta_{\max }=0.7$. 


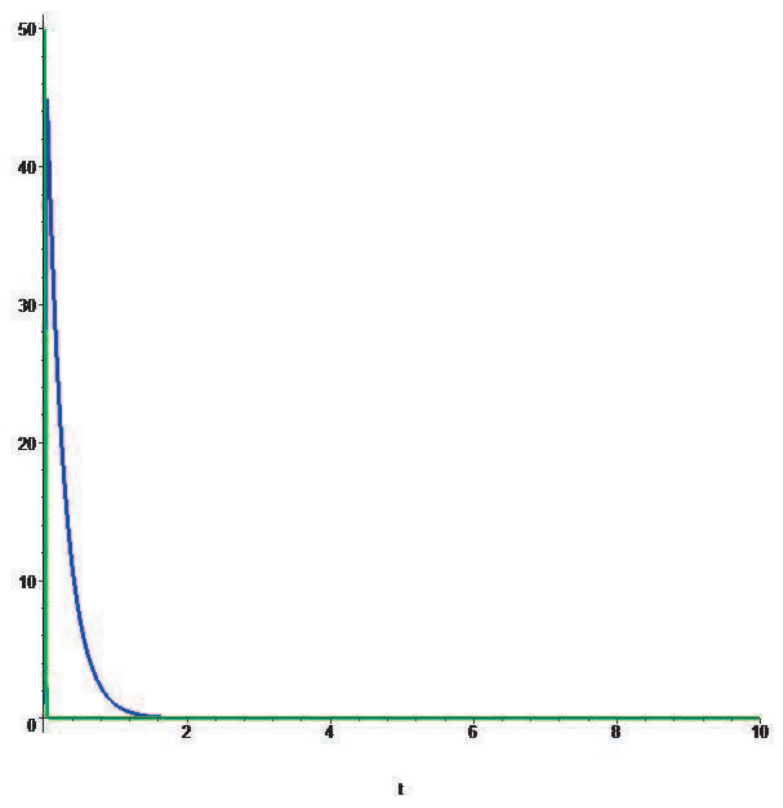

Figure 2. Case 4.14. Graphs of the optimal solutions $S_{*}(t), I_{*}(t)$ for $v_{\max }=4.0, \eta_{\max }=4.0$.

\section{Conclusions}

In this paper we have created and investigated a SIR type epidemic model of a population of constant size augmented by the four bounded controls: $p(t)$, vaccination of the newly born; $q(t)$, vaccination of all susceptible; $s(t)$, treatment of the infected; and $r(t)$, other indirect strategies. By the application of the Pontryagin Maximum Principle we stated and solved the optimal control problem of minimization of the cumulative number of the infected individuals on a given time interval $[0, T]$. The optimal controls were obtained analytically through qualitative investigation of the switching functions. It is demonstrated how parameters of the model influence the choice of the optimal policy. This work is unique in that multiple controls are mathematically analyzed jointly as opposed to the independent analysis single controls of an epidemic model that produces an optimal strategy of maximum effort on an initial time interval, i. e. at the beginning of epidemic $[4,31]$. In our model, when four controls are applied simultaneously, we obtained similar result for the three control functions that must be kept at maximum all the time. However, we also found that for some model parameters, the optimal control function $r(t)$, can have a piecewise constant form which implies that the implementation of precocious methods (quarantine, wearing masks and closing public events) can be delayed until a later time, $\theta_{*} \in[0, T)$. Numerical results illustrate the optimal solutions.

Acknowledgements. We are indebted to both of our anonymous reviewers for their useful comments. We would also like to thank the Editor, Dr. Natalie Hritonenko who have always been encouraging, helpful, and positive.

\section{References}

[1] R.M. Anderson, R.M. May. Infections Diseases of Humans: Dynamics and Control. Oxford University Press, Oxford, UK, 1992.

[2] S. Anita, V. Arnaǔtu, V. Capasso. An Introduction to Optimal Control Problems in Life Sciences and Economics. Birkhäuser, USA, 2011.

[3] N.T.J. Bailey. The Mathematical Theory of Epidemics. Griffin, London, 1957.

[4] H. Behncke. Optimal control of deterministic epidemics. Optim. Contr. Appl. Met., 21 (2000), No. 6, $269-285$. 
[5] A. Bressan, B. Piccoli. Introduction to the Mathematical Theory of Control. AIMS Series on Applied Mathematics, Vol. 2, AIMS, USA, 2007.

[6] V. Capasso. Mathematical Structures of Epidemical Systems. Lecture Notes in Biomathematics, Vol. 97, Springer, Heidelberg, 2008.

[7] C. Castilho. Optimal control of an epidemic through educational campaigns. Electron. J. Differential Equations, 2006 (2006), No. 125, 1-11.

[8] D.J. Daley, J. Gani. Epidemic Modelling: An Introduction. Cambridge University Press, Cambridge, 1999.

[9] B.P. Demidovich. Lectures on Stability Theory. Nauka, Moscow, 1967. (Russian)

[10] A.V. Dmitruk. A generalized estimate of the number of zeros for solutions of a class of linear differential equations. SIAM J. Control Optim., 30 (1992), No. 5, 1087-1091.

[11] K.R. Fister, S. Lenhart, J.S. McNally. Optimizing chemotherapy in an HIV model. Electron. J. Differentail Equations, 1998 (1998), No. 32, 1-12.

[12] E.V. Grigorieva, E.N. Khailov. Attainable set of a nonlinear controlled microeconomic model. J. Dyn. Control Syst., 11 (2005), No. 2, 157-176.

[13] E. Grigorieva, N. Bondarenko, E. Khailov, A. Korobeinikov. Finite-dimensional methods for optimal control of autothermal thermophilic aerobic digestion. In Industrial Waste, eds. K.Y. Show and X. Guo, Intech, Croatia, 2012, 91-120.

[14] E. Grigorieva, N. Bondarenko, E. Khailov, A. Korobeinikov. Analysis of optimal control problems for the process of wastewater biological treatment. Revista de Matemática: Teoría y Aplicaciones, 20 (2013), No. 2, $103-118$.

[15] E.V. Grigorieva, E.N. Khailov, A. Korobeinikov. Parametrization of the attainable set for a nonlinear control model of a biochemical process. Math. Biosci. Eng., 10 (2013), No. 4, 1067-1094.

[16] N.K. Gupta, R.E. Rink. Optimal control of epidemics. Math. Biosci., 18 (1973), 383-396.

[17] P. Hartman. Ordinary Differential Equations. John Wiley \& Sons, New York-London-Sydney, 1964.

[18] H.W. Hethcote, P. Waltman. Optimal vaccination schedules in a deterministic epidemic model. Math. Biosci., 18 (1973), 365-381.

[19] D.L. Jaquette. Mathematical models for controlling growing biological populations: a survey. Oper. Res., 20 (1972), $1142-1151$.

[20] H.R. Joshi. Optimal control of an HIV immunology model. Optim. Contr. Appl. Met., 23 (2002), No. 4, $199-213$.

[21] H.R. Joshi, S. Lenhart, M.Y. Li, L. Wang. Optimal control methods applied to disease models. In AMS Volume on Mathematical Studies on Human Disease Dynamics: Emerging Paradigms and Challenges, AMS Contemporary Mathematics Series, Vol. 410, 2006, 187-207.

[22] M.J. Keeling, P. Rohani. Modeling Infectious Diseases in Humans and Animals. Princeton University Press, Princeton, 2008.

[23] U. Ledzewicz, H. Schättler. On optimal sigular controls for a general SIR-model with vaccination and treatment. Discret. Contin. Dyn. S., supplement volume (2011), 981-990.

[24] E.B. Lee, L. Marcus. Foundations of Optimal Control Theory. John Wiley \& Sons, New York, 1967.

[25] S. Lenhart, J.T. Workman. Optimal Control Applied to Biological Models. CRC Press, Taylor \& Francis Group, London, 2007.

[26] R. Morton, K.H. Wickwire. On the optimal control of a deterministic epidemic. Adv. Appl. Probab., 6 (1974), No. 4, 622-635.

[27] L.S. Pontryagin, V.G. Boltyanskii, R.V. Gamkrelidze, E.F. Mishchenko. Mathematical Theory of Optimal Processes. John Wiley \& Sons, New York, 1962.

[28] R. Smith?. Modelling Disease Ecology with Mathematics. AIMS Series on Differential Equations \& Dynamical Systems, Vol. 2, AIMS, USA, 2008.

[29] A.N. Tikhonov, A.B. Vasil'eva, A.G. Sveshnikov. Differential Equations. Springer-Verlag, Berlin-Heidelberg-New York, 1985.

[30] F.P. Vasil'ev. Optimization Methods. Factorial Press, Moscow, 2002. (Russian)

[31] K.H. Wickwire. Mathematical models for the control of pests and infectious diseases: a survey. Theor. Popul. Biol., 11 (1977), No. 2, 182-238.

[32] G.S. Zaric, M.L. Brandeau. Resource allocation for epidemic control over short time horizons. Math. Biosci., 171 (2001), 33-58. 